\title{
Disjoint Decomposition for LUT FPGA Synthesis
}

\author{
Ulf Schlichtmann \\ Siemens AG, Semiconductor Group \\ 81549 Munich, Germany \\ Tel.: +49-89-4144-4823. Fax: +49-89-4144-8159. \\ email: ulf@hl.siemens.de
}

\begin{abstract}
Field-Programmable Gate Arrays (FPGAs) enjoy increasing popularity in the design of digital systems. It is imperative to support the design process with CAD tools if quality designs are to be obtained. However, the structure of FPGAs forbids the use of many algorithms developed for other types of chips. New algorithms are required. Decomposition techniques are especially well-suited for Look-Up-Table (LUT) FPGAs. This paper describes how to perform decompositions efficiently using ROBDDs. The quality of a function's decomposition depends on the selected partitioning of the function's variables. An effective heuristic to partition variables for decomposition is introduced in this paper.
\end{abstract}

\section{Keywords}

Functional decomposition, disjoint decomposition, ROBDD, variable partitioning

\section{INTRODUCTION}

Field Programmable Gate Arrays (FPGAs) are a class of integrated circuits that has seen rapid gains in usage and popularity in the recent past. Originally introduced about a decade ago, nowadays they are used extensively for prototyping and for production runs of small to medium volumes. The general architecture of all currently available FPGAs consists of a regular array of identical basic logic modules connected by routing resources. Both the logic module and the routing resources are user-programmable.

In Look-Up Table (LUT) FPGAs the logic module is dominated by a $2^{q}$ bit memory, thus allowing for the implementation of any Boolean function of at most $q$ inputs. The logic module is referred to as a q-LUT. Typical examples are the Xilinx FPGAs.

Logic synthesis and technology mapping are two key steps in the design of a digital circuit. The task of logic synthesis is to optimize a circuit, described by a set of Boolean equations. Generally, this optimization depends heavily on the identification of common subexpressions and on decomposition of Boolean functions into less complex functions (Brayton 1990). Traditionally, the quality of logic synthesis has been measured in the number of literals required to represent the circuit after optimization. The task of tech- 
nology mapping is to transform the optimized set of Boolean equations, usually represented as a Boolean network, into a netlist such that each element of the netlist can be implemented by a cell that is available in the given target technology.

Techniques for these tasks have been developed with the fine-grained cell libraries for mask-programmable ASICs in mind. While some of the techniques remain applicable for FPGAs, significant optimization potential would be wasted if these techniques were simply adapted for FPGAs. Instead, new techniques need to be developed.

Functional decomposition of Boolean functions is an optimization technique that is very effective both in logic synthesis and in technology mapping for LUT FPGAs. While the theory of functional decomposition of Boolean functions was originally developed about fourty years ago (Ashenhurst 1952), due to the high complexity of computing decompositions, it has not been put to use widely. However, recently is has been shown how to perform functional decompositions more efficient than previously possible, thus reviving interest in decomposition theory (Lai 1993b, Schlichtmann 1993). The quality of a functional decomposition depends on the selected partitioning of the function's variables. So far, no efficient partitioning strategies have been reported.

The organization of this paper is as follows. Following some definitions, Section 3 describes how to compute decompositions using ROBDDs. An effective heuristic to determine variable partitionings is introduced in Section 4. Results are given in Section 5.

\section{DEFINITIONS}

A Boolean variable $z$ can have two values, here denoted by 0 and 1 . By $\bar{z}$ the complement of the variable $z$ is denoted. Both $z$ and $\bar{z}$ are called literals.

Let $\underline{z}$ denote a vector of Boolean variables, $\underline{z}=\left(z_{1}, z_{2}, \ldots, z_{n}\right)$. With $|\underline{z}|$ the number of components of the vector is obtained. A fully specified, single output Boolean function, $F(\underline{z}): B^{|z|} \rightarrow B$ is a function of Boolean variables. A multi-output Boolean function is written as a vector $\underline{g}(\underline{z})$ of single-output Boolean functions. The number of outputs is obtained by $c=|g(\underline{z})|$. Alternatively, $\left(g_{1}(\underline{z}), \ldots, g_{c}(\underline{z})\right)$ will also be used instead of $\underline{g}(\underline{z})$. The number of elements in a set $S$ are obtained by $|S|$.

Reduced, Ordered Binary Decision Diagrams (ROBDDs) as proposed by Bryant (Bryant 1986) are employed. The expression $R O B D D(f)$ refers to the node $v$ that roots the ROBDD of $f$. By index $(v)$ the position of the variable associated with ROBDD-node $v$ in the variable ordering is obtained. To obtain the position of a variable $z$ in the variable ordering, index $(z)$ will be used as well. The first variable in the ordering is assigned index $=0$.

\section{DISJOINT DECOMPOSITION USING ROBDDS}

Given a Boolean function $F(\underline{z})$ and a partitioning of the variables into bound variables $\underline{x}$ and free variables $\underline{y}, F(\underline{z})=F(\underline{x}, \underline{y})$, an expression of $F$ having the structure $F(\underline{x}, \underline{y})=f(\underline{g}(\underline{x}), \underline{y})$ is termed a decomposition. The function $\underline{g}$ is called decomposition function, $f$ is called composition function. The value $c=|\underline{g}(\underline{x})|$ is referred to as the communication complexity of the decomposition. If no variables are shared between $\underline{x}$ and $\underline{y}$, the decomposition is said to be disjoint, otherwise non-disjoint. This paper focusses 


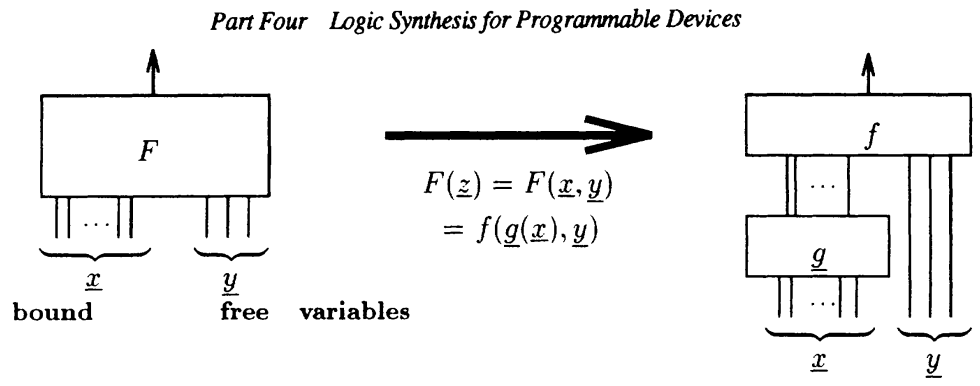

Figure 1 Structure of a disjoint decomposition

on disjoint decompositions. The structure of such decomposition is shown graphically in Figure 1. The decomposition problem is: Given $F(\underline{z})$ and a partitioning of $\underline{z}$ into $\underline{x}$ and $\underline{y}$, determine the minimum communication complexity.

Previously, methods have been suggested to determine $g(\underline{x})$ based on decomposition charts (Ashenhurst 1952) and on the on- and off-covers of Boolean functions (Roth 1962). While these methods solve the decomposition problem, they are not as efficient as the ROBDD-based approach discussed next.

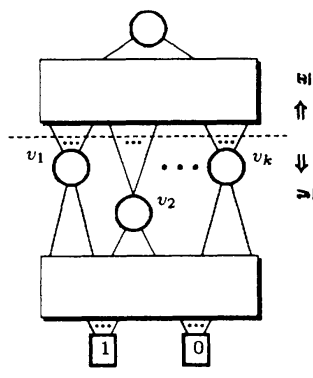

a)

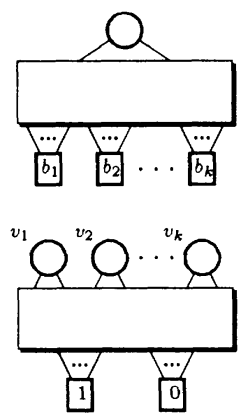

b)

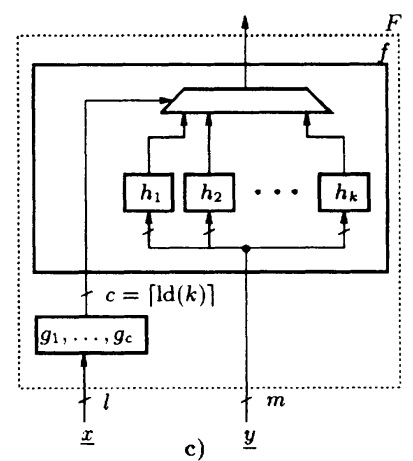

c) $\underline{y}$

Figure 2 Computing a disjoint decomposition using ROBDDs

The basic idea of the ROBDD-based approach is shown in Figure 2. An ROBDD is built for function $F(\underline{x}, y)$. The bound variables are arranged before the free variables in the variable ordering. Assume $l=|\underline{x}|$. If a cut-line is drawn at level $l$ in the ROBDD, i.e. separating the BDD-nodes $v_{b}$ with index $\left(v_{b}\right)<l$ from the nodes $v_{f}$ with index $\left(v_{f}\right) \geq l$, then cut_nodes $(R O B D D(F(\underline{x}, \underline{y}), l))$ denotes the set of nodes that are located below the cut-line and have at least one incoming edge that originates above the cut-line. The ROBDD is then cut at the position of the cut-line. Each of the nodes $v_{1}, \ldots, v_{k}$ in this set now becomes a root node of an ROBDD. The functions $h^{1}(\underline{y}), \ldots, h^{k}(\underline{y})$ rep- 
resented by ROBDDs rooted by $v_{1}, \ldots, v_{k}$ only depend on the free variables $y$. They can be thought of as being connected to the data inputs of a $k$-to-1 MUX. Thus, the communication complexity $c$ is easily determined from a function's ROBDD. Then $c=\lceil l d k\rceil$ $=\left\lceil\mid c u t \_n o d e s(\operatorname{ROBD} D(F(\underline{x}, \underline{y}), l)) \|\right.$ control inputs are needed to select one of the data inputs. The functions $g(\underline{x})$ computing these control inputs only depend on the bound variables $\underline{x}$. This way, in Figure $2 c$, the decomposed structure shown on the right-hand side in Figure 1 is obtained.

Computing a decomposition using an ROBDD results in speedups ranging from 17 to 35 compared to previously known methods on a set of benchmark examples (Lai 1993b).

\section{PARTITIONING VARIABLES TO MINIMIZE COMMUNICATION COMPLEXITY}

The problem of selecting a variable partitioning has received only little attention so far. In (Lai 1993) all partitions with $|\underline{x}|=l(l=4$ or 5$)$ are enumerated and evaluated. This approach can be quite costly for Boolean functions that depend on many variables. Additionally, since $|\underline{x}|$ is constrained, only a limited solution space is evaluated. It may instead be desirable in the first steps to decompose a large function into intermediate functions that themselves are still too large to fit into a given $q$-LUT. In (Sasao 1993) only decompositions with $|y|=2$ are considered, thus significantly limiting the solution space. In (Schlichtmann 1993) it is shown that it is beneficial to select symmetric variables as bound variables. Many Boolean functions, however do not have symmetric variables.

Therefore, a heuristic is presented here to adequately address the problem of determining an effective partitioning. It proceeds by first determining an initial solution followed by an iterative improvement phase. These steps result in a variable ordering. Only then a cut in the ordering is selected to obtain the final variable partitioning. A cost function is presented that guides this selection of the cut position. No restriction is placed on the number of variables in either bound set or free set.

\subsection{Initial Variable Ordering}

This heuristic is based on the assumption that generally an ROBDD that is small in the number of nodes will also allow decompositions with a small communication complexity. It proceeds as follows:

1. Search for a variable $z_{d}$ such that $F\left(z_{1}, \ldots, z_{d}=1, \ldots, z_{n}\right)=1$, i.e. $z_{d}$ can be disjunctively split from $F(\underline{z})$. If found, delete this variable from $F(\underline{z})$, remember it and continue with step 1 .

2. Search for a variable $z_{c}$ such that $F\left(z_{1}, \ldots, z_{c}=0, \ldots, z_{n}\right)=0$, i.e. $z_{c}$ can be conjunctively split from $F(\underline{z})$. If found, delete this variable from $F(\underline{z})$, remember it and continue with step 1 .

3. Arrange the variables found so far in reverse order at the end of the variable ordering. The variable found first will be the last variable and so on.

4. On the remaining function, apply the procedure reported by $\mathrm{Wu}$ and Marek-Sadowska (Wu 1993). 


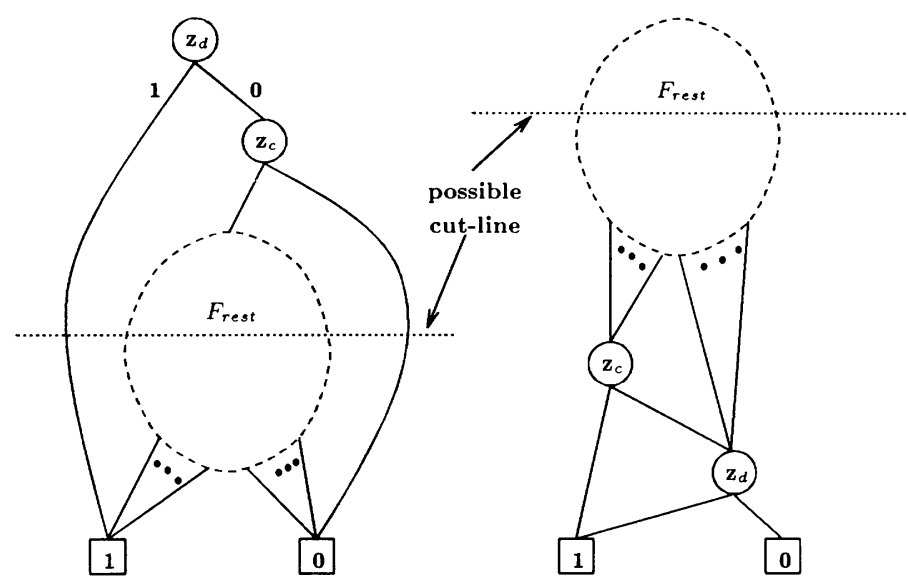

Figure 3 Ordering of variables that can be split off disjunctively or conjunctively

The rationale for step 3 in the above procedure is illustrated in Figure 3 for the ROBDD of a function $F(\underline{z})=z_{d}+z_{c} \cdot\left(F_{\text {rest }}\left(\underline{z}^{\prime}\right)\right)$ where $\underline{z}^{\prime}$ does not contain $z_{d}$ and $z_{c}$ anymore. If the variables $z_{d}, z_{c}$ were put at the top of the variable ordering as shown in the left part of the Figure, the 1-successor of $z_{d}$ would lead directly to the terminal node 1, the 0 -successor of $z_{c}$ would lead directly to the terminal node 0 . Then, for every cut-line below $z_{c}$ both terminal nodes would be contained in the set cut_nodes. If the variables are put into the last position of the variable ordering, as shown in the right part of the Figure, this problem is remedied. The ROBDD of $F_{\text {rest }}$ (dashed in the Figure) remains unchanged.

\subsection{Iterative Improvement}

The variable ordering so obtained will usually not represent an optimum. Therefore, an iterative improvement heuristic is employed next.

The basic idea of the method is to swap variables across a given cut-line. Whereas up to now variable orderings have been considered without regard to a specific location of a cut-line, now variables need to be swapped with regard to a specific cut-line. However, the best location for the cut-line can only be determined after iterative improvements of the variable ordering have been performed. Therefore, variable swapping needs to be performed for every possible cut-line. Thus now $|\underline{z}|-1$ ROBDDs will be considered, one for each possible cut-line.

The algorithm variable_exchange is shown in Figure 4. The cut-lines are considered sequentially. The level(cut) of cut is the minimum index $(v)$ of any node $v$ located below cut. $R O B B D^{l}(F(\underline{z}))$ denotes the ROBDD of $F(\underline{z})$ that is being treated with respect to $c u t$ with $l=$ level $(c u t)$. ROBD $D_{z_{u} \leftrightarrow z_{l}}(F(\underline{z}))$ denotes the ROBDD with variables $z_{u}$ and $z_{l}$ exchanged in the variable ordering. Variables are always swapped in pairs, i.e. for cut at level $l$ a variable $z_{u}$ with $\operatorname{index}\left(z_{u}\right)<l$ is swapped with another variable $z_{l}$ 


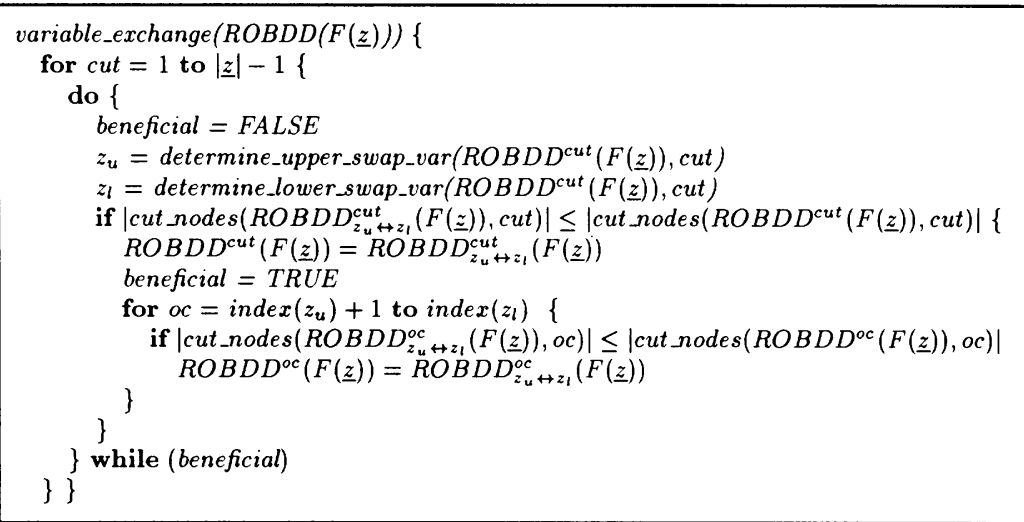

Figure 4 Iterative improvement of variable ordering based on variable exchanges

with index $\left(z_{l}\right) \geq l$ in the variable ordering. A swap is considered to be beneficial, if it decreases $\mid$ cut_nodes $(R O B D D(\underline{z}), l) \mid$. The communication complexity is obtained easily as $\left\lceil l d\left(\left|c u t \_n o d e s(R O B D D(\underline{z}), l)\right|\right)\right\rceil$. For a given $c u t$ at level $l$, variable swaps are performed as long as beneficial swaps exist. Pairs of swap variables are determined by tentatively swapping each variable $z_{u}$, index $\left(z_{u}\right)<l$ below cut and selecting the variable that yields an optimum of $\mid$ cut_nodes $(R O B D D(\underline{z}), l) \mid$. This is done by determine_upper_swap_var. The respective procedure is performed for all variables $z_{l}$, index $\left(z_{l}\right) \geq l$, by swapping them above cut. The best variables are then permanently swapped, if the swap is beneficial.

To consider more than just one cut-line at a time, after each swap of $z_{u}$ and $z_{l}$ it is checked if this swap would improve the solution for other cut-lines also. Only other cut lines $o c$ in the range index $\left(z_{u}\right)<\operatorname{level}(\mathrm{oc}) \leq \operatorname{index}\left(z_{l}\right)$ need to be considered.

\subsection{Determining Partitioning}

Now the variable ordering has been improved for every possible cut-line. In order to actually perform the decomposition, one of the possible cut-lines needs to be selected to determine a partitioning of $\underline{z}$ into $\underline{x}$ and $\underline{y}$.

To this end, a cost function is proposed that estimates the number of logic modules required to implement a Boolean function $f$ in $q$-LUTs. It is based on the assumption that a function will be decomposed according to the Shannon expansion $f(\underline{z})=z_{i} \cdot f(\underline{z})+$ $\bar{z}_{i} \cdot f(\underline{z})$. While this leads to a pessimistic estimation of implementation cost, empirically it has yielded good results in guiding the choice of a partioning of the variables.

The cost of implementing a single-output function $g_{i}(\underline{x})$ is given as:

$$
\operatorname{cost}(\underline{g}(\underline{x}))= \begin{cases}1 & \text { for }|\underline{x}| \leq q \\ 2^{(|\underline{x}|-q)+1}-1 & \text { for }|\underline{x}|>q\end{cases}
$$


The total cost $C$ of a decomposition of a function $F(\underline{x}, \underline{y})=f(\underline{g}(\underline{x}), \underline{y})$ then is

$C=\operatorname{cost}(f(\underline{g}(\underline{x}), \underline{y}))+\sum_{i=1}^{|\underline{g}(\underline{x})|} \operatorname{cost}\left(g_{i}(\underline{x})\right)$

Of all possible variable orderings associated with different cut-lines cut, the cut-line that results in a decomposition minimizing $C$ is selected.

\section{RESULTS FOR TECHNOLOGY MAPPING ON XILINX}

The presented decomposition method has been implemented in the synthesis tool TOS. It is embedded into a design flow that consists of preprocessing the initial Boolean network by assuring that no two nodes in network compute the same function, either globally or locally. A partial collapsing is performed where nodes are collapsed into their successors, if these will then still have no more than $q$ fanins. After decomposition, the same operations are performed as a postprocessing step.

Finally, nodes in the network are merged into logic modules (Configurable Logic Blocks, CLBs) as permitted by the technology, using cardinality matching.

\begin{tabular}{rrrrr}
\hline & \multicolumn{2}{c}{ Random } & \multicolumn{2}{c}{ BDD-Min } \\
& CLBs & CPU [s] & CLBs & CPU [s] \\
\hline NO iterative improvement & 2927 & 57.2 & 1774 & 27.9 \\
\hline WITH iterative improvement & 1773 & 113.8 & 1715 & 72.9 \\
\hline
\end{tabular}

Table 1 Effects of various options of the presented algorithm

Table 1 permits an evaluation of the presented methods for variable partitioning. TOS optimized for number of CLBs on the XC3000 architecture. The total number of required CLBs for a set of $20 \mathrm{MCNC}$ benchmark circuits is given. Four data points are presented. Variables were ordered both randomly (Random) and using the heuristic presented in Section 4.1 (BDD-Min). Additionally, the iterative heuristic of Section 4.2 was employed to improve on the initial results.

Two facts are obvious from Table 1: the iterative improvement heuristic is very effective. The better it's starting situation, the better it's final results. Also, the heuristic proposed to select an initial variable ordering is very effective. Just by itself it results in almost exactly the same number of required CLBs as using the iterative improvement heuristic on a random variable ordering.

The required CPU time increases by a factor of 2 to 3 when the iterative improvement heuristic is used. Interestingly, using the BDD-Min heuristic results in less CPU time than using a random ordering. This is because with a random ordering, the number of decompositions that need to be performed rises significantly. 


\section{CONCLUSION AND FUTURE WORK}

A method to compute disjoint decomposition using ROBDDs has been presented. Also, an effective method to partition variables for decomposition has been introduced. It has been shown that these methods yield good results using only little CPU time.

Future work will focus on non-disjoint decomposition, decomposition of multi-output Boolean function and improved methods for variable partitioning.

\section{REFERENCES}

Ashenhurst, R. L. (1952) The Decomposition of Switching Functions. Bell Laboratories' Report No. BL-1.

R. K. Brayton, R. K.; Hachtel, G. D. and Sangiovanni-Vincentelli, A. L. (1990) Multilevel Logic Synthesis. Proceedings of the IEEE, 78, $264-300$.

Bryant, R. E. (1986) Graph-Based Algorithms for Boolean Function Manipulation. IEEE Transactions on Computers, 35, 677 - 91.

Lai, Y.-T.; Pan, K.-R. R.; Pedram, M. and Sastry, S. (1993) FGMap: A Technology Mapping Algorithm for Look-Up Table Type FPGAs Based on Function Graphs. Workshop Notes International Workshop on Logic Synthesis IWLS, 9b1 - 4.

Lai, Y.-T.; Pedram, M. and Vrudhula, S. B. K. (1993b) BDD Based Decomposition of Logic Functions with Applications to FPGA Synthesis. 30th Design Automation Conference $D A C, 642-7$.

Roth, J. P. and Karp, R. M. (1962) Minimization over Boolean Graphs. IBM Journal of Research and Development 6, 227 - 38.

Sasao, T. (1993) FPGA Design by Generalized Functional Decomposition, in Logic Synthesis and Optimization (ed. T. Sasao), Kluwer Academic Publishers, Boston / London / Dordrecht.

Schlichtmann, U. (1993) Boolean Matching and Disjoint Decomposition for FPGA Technology Mapping. Proceedings IFIP International Workshop on Logic and Architecture Synthesis, $83-102$.

Wu, Y.-L. and Marek-Sadowska, M. (1993) Efficient Ordered Binary Decision Diagram Minimization Based on Heuristics of Cover Pattern Processing. European Conference on Design Automation EDAC, $273-7$.

\section{BIOGRAPHY}

Ulf Schlichtmann joined the Institute of Electronic Design Automation (Prof. Dr.-Ing. K. Antreich) in 1990 to start the synthesis research group. Together with Michael Hermann he laid the foundation for the FPGA synthesis tool TOS. In 1991, he spent 2 months as a visiting scholar with the Microelectronics Center of North Carolina, Research Triangle Park, USA. His dissertation focusses on core algorithms for FPGA oriented logic synthesis and technology mapping. Since 1994, he is a member of the technical staff in the Siemens semiconductor group, mainly involved with macrocell based IC design and design reuse. 